\title{
Acute Suppurative Thyroiditis
}

Parth Desai, DO; Chimezie Mbachi, MD; Anshu Singh, MBBS, MD

From the Department of Internal Medicine at John H. Stroger, Jr. Hospital of Cook County in Chicago, Illinois.

Financial Disclosures: None reported

Support: None reported

Address correspondence to

Parth Desai, DO, John

H. Stroger, Jr. Hospital of Cook County, 1969 Ogden Ave, Chicago, IL 60612-3765. Email: parth.desai@ cookcountyhhs.org

Submitted June 3, 2020; revision received

July 8, 2020;

accepted July 23,2020

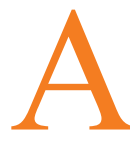

25-year-old woman presented to the emergency department with a 2-week

history of progressively increasing neck swelling associated with pain, dysphagia, and orthopnea. On examination, hervitals were normal. A fluctuant, tender $3.4 \mathrm{~cm}$ mass was palpated in the right thyroid gland. Contrast-enhanced computed tomography of the neck showed marked enlargement of the thyroid lobe and the lateral aspect of the thyroid isthmus with diffusely decreased attenuation, multiple enhancing septations, and inflammatory changes in the surrounding soft tissues (image A, red arrow). The trachea was mildly displaced to the left (image B, blue arrow). The patient was treated with IV ampicillinsulbactam and incision and drainage due to multiple septations, making adequate drainage with fine-needle aspiration difficult. Fluid cultures grew Streptococcus anginosus. The patient was stable upon posthospital follow-up with no further neck swelling.

Acute suppurative thyroiditis (AST) is rare and commonly caused by Staphylococcus aureus, Streptococcus pyogenes, Staphylococcus epidermidis, and Streptococcus pneumoniae. ${ }^{1}$ Streptococcus anginosus is part of human oral flora and a known cause of head and neck abscesses, which may cause AST through local extension. ${ }^{1,2}$ Patients present with anterior neck swelling and fevers and may develop transient thyrotoxicosis with subsequent hypothyroidism if left untreated. ${ }^{1,3}$ AST may be associated with anatomic abnormalities such as pyriform sinus fistula, necessitating repeat anatomic imaging after recovery of the acute infection. ${ }^{3,4}$ In cases without airway compromise, management includes fineneedle aspiration for drainage and culture-directed antibiotic therapy. If the airway is compromised, patients may require immediate operative management. ${ }^{2}$ Prompt identification and management of the condition is paramount, as cases may be fatal. $^{4}$ (doi:10.7556/jaoa.2020.132)

\section{References}

1. Wu C, Zhang $Y$, Gong $Y$, et al. Two cases of bacterial suppurative thyroiditis caused by Streptococcus anginosus. Endocr Pathol. 2013;24(1):49-53. doi:10.1007/ s12022-013-9236-0

2. Noguchi S, Yatera K, Kawanami T, et al. The clinical features of respiratory infections caused by the Streptococcus anginosus group. BMC Pulm Med. 2015;15:133. doi:10.1186/ s12890-015-0128-6

3. Paes JE, Burman KD, Cohen J, et al. Acute bacterial suppurative thyroiditis: a clinical review and expert opinion. Thyroid. 2010;20 (3):247-255. doi:10.1089/thy.2008.0146

4. Falhammar $\mathrm{H}$, Wallin $\mathrm{G}$, Calissendorff J. Acute suppurative thyroiditis with thyroid abscess in adults: clinical presentation, treatment and outcomes. BMC Endocr Disord. 2019;19(1):130 doi:10.1186/s12902-019-0458-0

(๑) 2020 American Osteopathic Association 


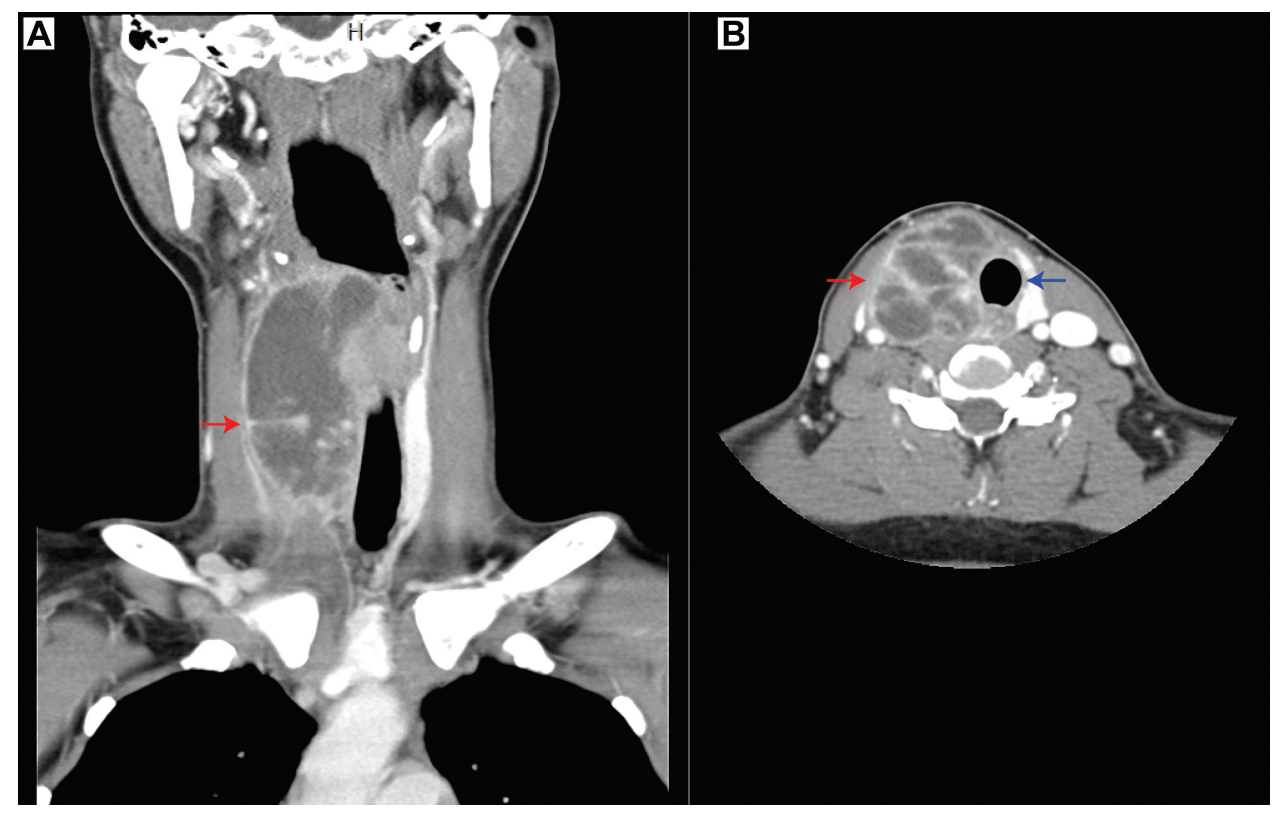

